\title{
Analysis and Design of University Scientific Research Management System Based on UML
}

\author{
Shidan $\mathrm{He}^{1}$ \\ Xijing University, 710123, Xi'an, China \\ e-mail: 315705804@qq.com
}

\begin{abstract}
UML is a language for the specification, construction, visualization and documentation of the software system. In this paper, the UML method is used to analyze and design the scientific research management system in colleges and universities, and to establish a system model which is based on scientific research management as the center of the system structure and the $B / S$ structure.
\end{abstract}

Keywords-Research management; System model; The B/S structure; $U M L$

\section{PROJECT BACKGROUND}

Scientific research activities and scientific research ability of universities have become an important indicator to measure the comprehensive strength of colleges and universities[1]. But at present, the majority of the scientific research management work of universities mainly stays in WEB site of a simple release stage to a great degree and mainly relies on manual to deal with a large number of data, which does not only bring a heavy workload to the scientific management personnel and the lack of communication between researchers and scientific research staff will resultin slow flow, thereby affect the quality of scientific research management.

The establishment of a good university scientific research project management system is to realize the digital management of university scientific research, improve the work efficiency of scientific research and personnel business management, speed up the record, access and transmission speed of information. In this paper, the UML method is used to analyze and design the management system of scientific research projects in universities and to develop a dynamic research data center and scientific research management communication platform, which can realize the network management of scientific research work[2].

\section{DEMAND ANALYSIS OF UNIVERSITY SCIENTIFIC RESEARCH MANAGEMENT SYSTEM}

University scientific research management work mainly includes information management of scientific researchers, research project, scientific and technological achievements management, intellectual property management and project management, etc., is a complex and frequent operation. According to different business categories, it can be divided into many kinds of business personnel, such as scientific research personnel, scientific research management, scientific research management personnel, school leader guide, department of management, and so on[3]. Various aspects of scientific research

\author{
Xiaofang $\mathrm{Li}^{2}$ \\ Xijing University, 710123, Xi'an, China \\ e-mail: lixiaofangxijing@126.com
}

management work around is mainly divided into different business people in an orderly manner, such as management of scientific research project reporting, assessment, project, and the end of the process, the need for scientific research and management of the organization and management of scientific research staff to responsible for business reporting, business acceptance, project reporting organization, etc..

Along with the rapid development of school research, most of the school's release function single obviously cannot meet the needs of current scientific research and management. It is urgently needed to establish a new scientific research management platform, to improve the efficiency of scientific research management through increasing the distribution management design, communicating timely and other functions. In order to meet the needs of scientific research management, the function of university scientific research management system is composed of five functional modules, such as my research, personnel management, information dissemination, project reporting, system security, etc. [4], as it is depicted in Figure 1:

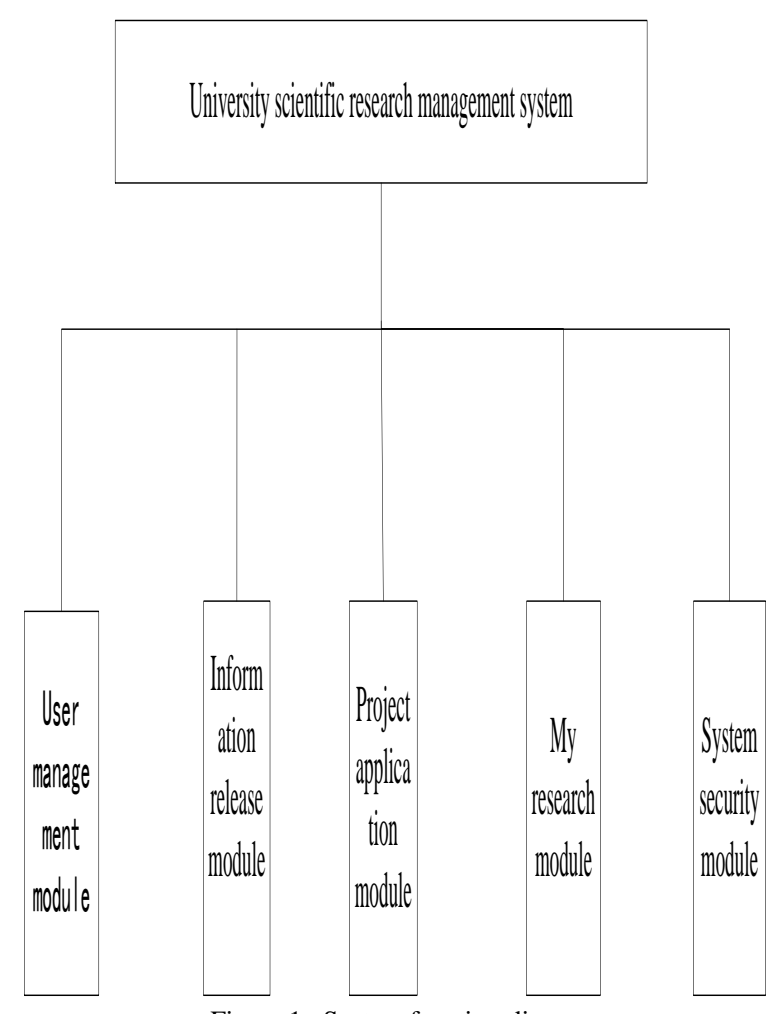

Figure 1. System function diagram 


\section{SYSTEM MODELING BASED ON UML}

UML is a language for the specification, construction, visualization, and documentation of the software system. The purpose of analysis and design system is to produce a usable and complete solution based on UML, and it can be easily converted into program code, which can be divided into business modeling and requirement analysis. design model, system implementation, testing and system configuration. The modeling of university research management platform should be based on the needs of existing business, and take full account of the practical applicability of the design. In the process of development, with the deepening of the understanding of the system, the establishment of the model achieves top-down continuous expansion and refinement, analysis stage sets the semantic category which is further extended to define new classes to deal with technical problems, thus form a practical platform for the university scientific research management UML model[5].

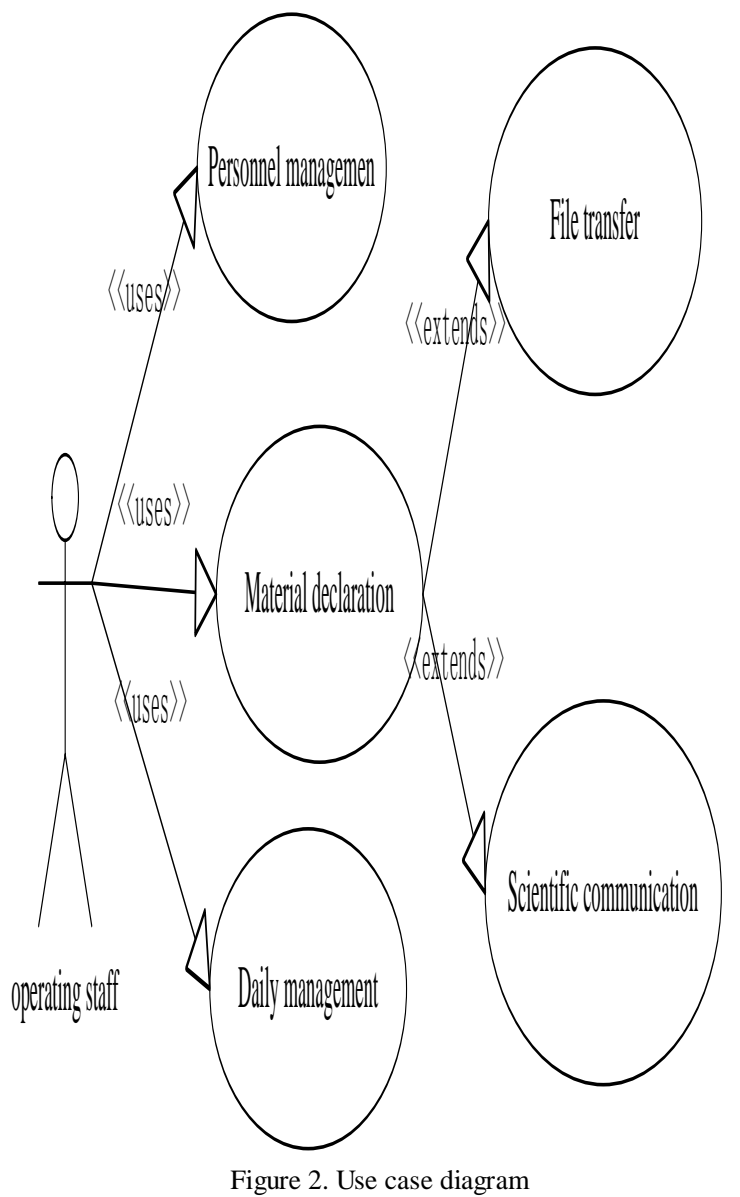

\section{1) System requirements use case modeling}

In the early stage of the system design, the main functions of the research staff in the platform can be considered as the scientific research management for the management of the personnel, the scientific research project, the publication of the daily information and the exchange of scientific research. Through the analysis of system requirements, in the system, it can be identified as the major players from system access points: General browsing, intramural scientific research personnel, research Secretary of college, scientific research and management personnel, university decision makers, system administrators role using this system according to their different permissions[6].

\begin{tabular}{|l|}
\hline BLL::Domain::Model:FindStatistics \\
\hline -TimerRange \\
-MaterialName \\
-MaterialShop \\
-MaterialDiffer \\
\hline \\
\hline
\end{tabular}

Figure 3. Data access object class

2) Static structure analysis and design of scientific research management system

For further analysis of system requirements, the first is to determine the system's static structure. The system's static structure model is mainly used to describe the class diagram. Class diagram design is the core part of the system design, it is a general basis for the UML and other modeling tools to generate code, to help analysis and design of the development of the follow-up greatly. The class diagram does not only describe the visual modeling language, but also represents a variety of relationships between the classes, showing the whole system of the static structure. After the analysis of the model of scientific research management, it can be concluded that there are at least 4 solid bodies in the system, User, Information, Record and Program. In order to facilitate data access, the data access object classes for each entity class are designed. Table 1 is the description of the real body[7].

In order to facilitate data access, it is necessary to design data access object class for each entity class. Figure 3 is NameDao. FindID method is based on the number of users to find a household survey. Update () method updates user information. In order to complete the operation of the user's business logic, the system is required to set up a service class NameService, as shown in Figure 4. ValidationCode () method is used to generate the verification code, and Nameacess () method is used to verify the existence of the account.

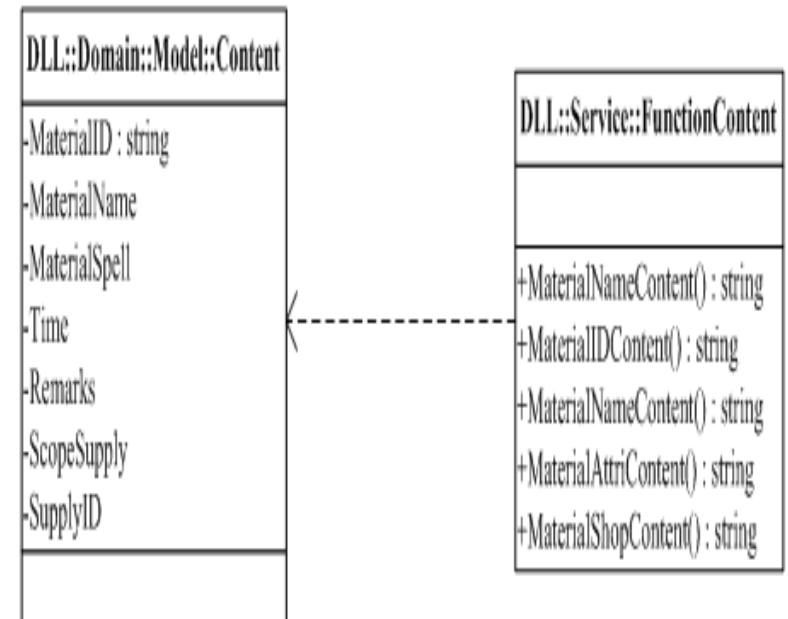

Figure 4. Class diagram 


\section{3) Dynamic structural analysis and design of scientific research management system.}

The system needs to consider the various processes and activities of the user to use the system to carry out scientific research management, which involves different users' rights. Therefore, the user's activities are also different. UML dynamic model describes all aspects of the system dynamic behavior, including the use case diagram, sequence diagram, collaboration diagram, activity diagram and state diagram. Figure 5 depicts the user declare the activity of scientific research projects, display the user declare the project of scientific research more intuitively[8].

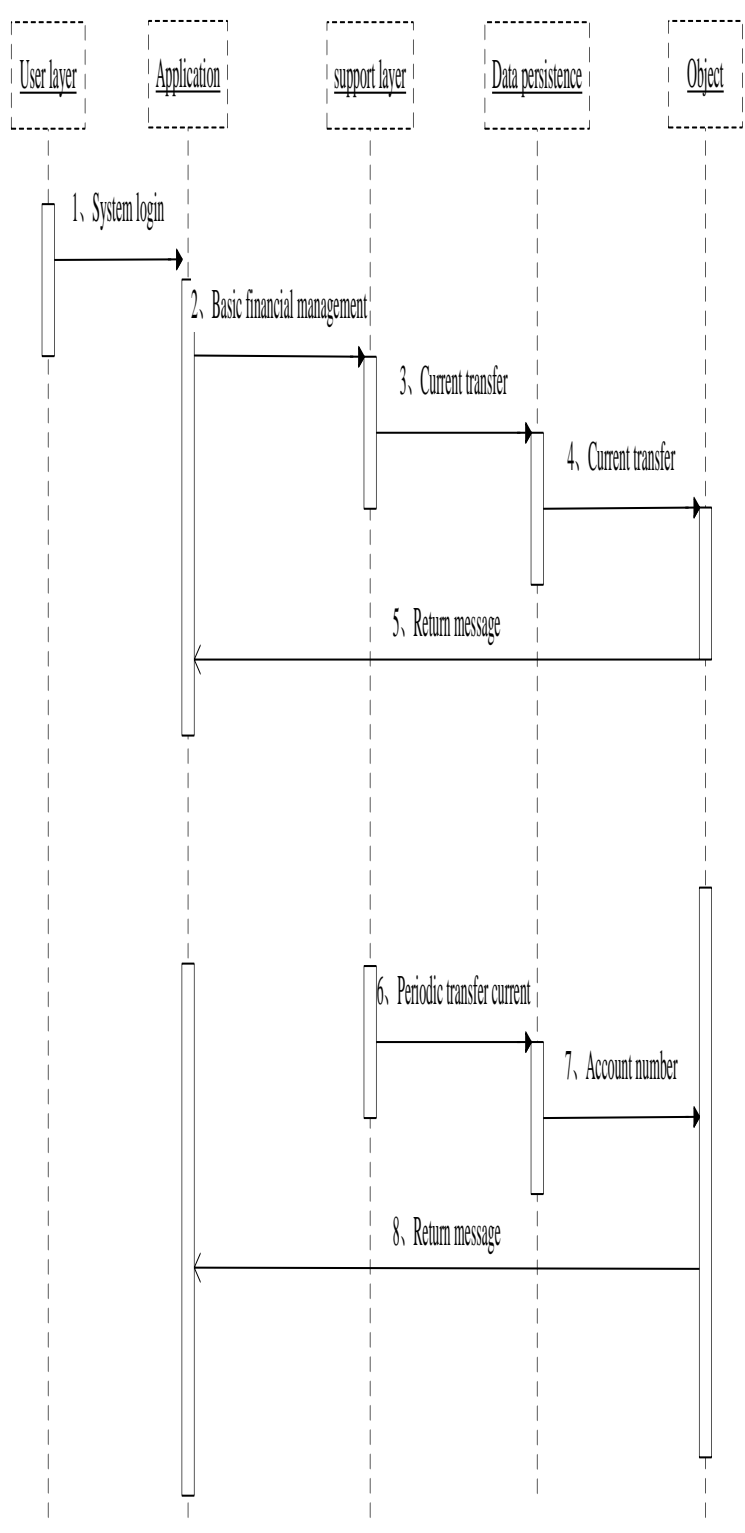

Figure 5. Time sequence diagram

In UML diagrams, sequence diagrams are said as a two-dimensional diagram, which describes the transmission of time sequence of messages among objects where the vertical axis for the time axis (such as the dotted line in Figure 6), the horizontal axis is for the cooperation in each independent object. Figure 6 is for the scientific research personnel to carry out the order of the project[9].

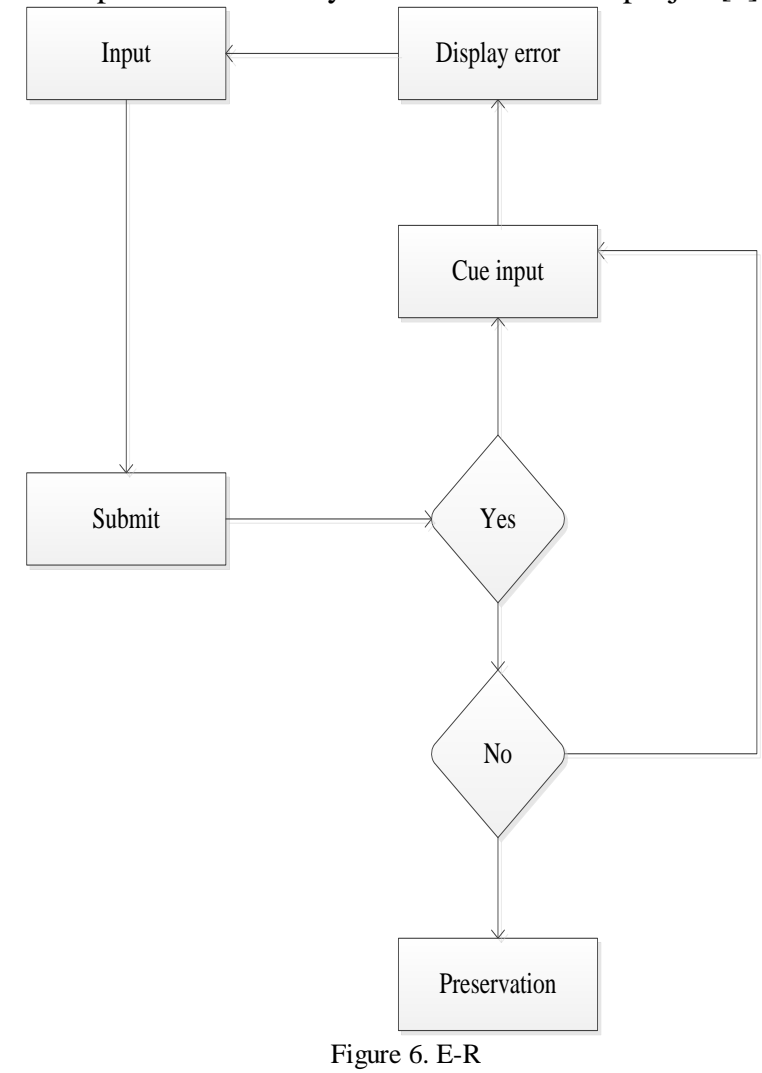

\section{SYSTEM DEVELOPMENT AND IMPLEMENTATION}

The system analyzes and designs the object oriented modeling and analysis, useing UML and modeling tool which is on the basis of the analysis of university scientific research management system. It can reduce the complexity of the system and improve the quality of software development and efficiency. After the analysis and design of the system, the system takes the security, versatility and scalability of scientific research management systeminto account. The Net technology is the core, and the post station is collected with Microsoft's Server SQL system, which is based on $\mathrm{B} / \mathrm{S}$ mode. A university scientific research management system has been developed to meet the needs of daily needs[10].

\section{CONCLUSION}

UML is a language for building model, which is the object-oriented visual system analysis. The various models designed through the analysis of university scientific research management system in the UML method can help scientific research to understand the university management business process better, establish a more reliable and more perfect system model. So that the users and developers can achieve the same understanding about the problem description, in order to reduce the differences of semantic and to ensure the correctness of the analysis.

\section{ACKNOWLEDGEMENTS}

This work is supported by Natural Science Foundation of Xijing University(No. XJ140209). 


\section{REFERENCES}

[1] Ningsheng Guo, Dongbo Wang, Yangxi Fan. Analysis and Design of Digital Drawing Management Information System based on UML.Mechanical Science and Technology for Aerospace Engineering; Vol. 28 No 3,2009, p. 404-11

[2] Martin Fowler. UML Distilled, Beijing:Tsinghua University Press ;2005

[3] Limin Zhang, Hongxia Xia. School Administration Management Information System in University Based on Um, Journal of Wuhan University of Technology; Vol.31 No.12,2009, p. 139-42

[4] Jun Yang.The Design of the Project Information Manage System Based on UML,Computer Knowledge and Technology;Vol.6 No.23,2010, p. 6397-9

[5] Liang Xiong, Manshan Lin. Analysis and Design of Civil Aid Management Informations System Based on UML, Journal of Beijing Elrctron in Science and Technology Institute; Vol.18, No.2 2010,p. 91-6
[6] Xiaofeng Luo, Wanbo Luo, Yue Hu.A Study on Governance of Net Public Sentiments.Communications Technolog;No.04,2010, p. 81

[7] Yan Gao:The Introduction for Police Involved Emergencies,Jounrnal of Chinese Peoples Public Security University(Social Sciences Edition);No.14,2010, p. 47-50

[8] Ming Chen: Software Engineering,Beijing: publishing House of Electronics Industry;2004

[9] Qixiong Dong, Ying Gong and Bo Li:Design of Voting System Based on Three-tier Architecure,Information \& SeienceElectronics Industr;2010, p. 11

[10] A. Rosenthal, P. Mork, M.H. Li, J. Stanford, D. Koester, P. Reynolds Cloud computing: a new business paradigm for biomedical information sharing J. Biomed. Inform., 43 (2010), pp. $342-353$ 